\title{
Urinary diversion: historical aspect and patient's satisfaction
}

\begin{abstract}
During the last decades, the techniques of urinary diversion have evolved from simple diverting techniques through a conduit to the orthotopic neobladder reconstruction aiming to provide an improved quality of life for patients undergoing radical cystectomy for muscle-invasive bladder cancer. The outcome after cystectomy and continent urinary diversion is not only dependent on surgical technique but restrictive preoperative patient selection and expert perioperative patient management is of equal importance. Urinary diversion techniques are usually limited by two main factors; patient or medical criteria, or both. Important patient criteria are patient's preference, age and co morbidity, body mass index (BMI) and motivation. Medical criteria for choice of the type of urinary diversion are renal function, upper urinary tract status, underlying primary disease, indication for cystectomy and limitations concerning the gastrointestinal tract. Complications after urinary diversion are generally due to the surgical technique, the underlying disease process and its treatment, patient's, comorbidities and the follow-up period. Patient counseling for better satisfaction about the type and technique of urinary diversion should be tailored to determine the safest method for cancer control, with the least complications along both the short and the long terms, and the easiest comfortable adjustment for a better patients' lifestyle, to obtain the best patients' quality of life. Health-related quality of life (HRQoL) measures should include the patient's physical fitness, the underlying symptoms, global judgment of health, psychological and social well-being, cognitive function, and personal constructs.
\end{abstract}

Keywords: urinary diversion, historical, patient's satisfaction
Volume I Issue 3 - 2014

\begin{abstract}
Aly Saber
Department of general surgery, Port Fouad General Hospital, Egypt
\end{abstract}

Correspondence: Aly Saber, Department of general surgery,
Port Fouad General Hospital, 19 al-guish street, Port Fouad, Port Said, Egypt, Email alysaber54@gmail.com

Received: October 22, 2014 | Published: December 30, 2014
Abbreviations: BMI, body mass index; HRQoL, health-related quality of life; WHO, world health organization; SIU, society internationale d'urologie; QoL, quality of life; FACT-BL, functional assessment of cancer therapy-bladder cancer; HADS, hospital anxiety and depression scale

\section{Historical aspect}

During the last decades, the techniques of urinary diversion after radical cystectomy for muscle-invasive bladder cancer have evolved from simple diverting techniques through a conduit to the orthotopic neobladder reconstruction thus providing a safe and continent reservoir to store and eliminate urine with efforts to provide an improved quality of life. ${ }^{1}$ Sir John Simon is credited with performing the first ureteroproctostomy for bladder exstrophy in 1851. Simon attempted ureterosigmoidostomies in dogs by bringing the ureters into intimate contact with the rectum below the peritoneal reflection in order to create a fistula using silk sutures. Subsequently, Lloyd attempted a similar technique in an exstrophy patient resulting in peritonitis and death at the $7^{\text {th }}$ postoperative day. ${ }^{2}$ In 1879 , Thomas Smith utilized a two layer technique with direct anastomosis of ureteral mucosa to bowel mucosa with a second line of sutures used to close the sero muscular layers of the bowel around the ureter. In 1892, Karl Maydl tried to decrease the high incidence of uretero-intestinal strictures by implanting an ellipse of trigone carrying both ureteric orifices into the sigmoid colo. ${ }^{2}$ In 1907 and 1909, Ricard succeeded to introduce the split-cuff nipple method of uretero vesical and uretero intestinal anastomoses and in 1922, Robert C. Coffey introduced an anti reflux technique for anastomosing the ureter to the caecum in dogs. After that, Nesbit described a direct mucosa-to-mucosa anastomosis of spatulated ureter to the sigmoid colon using the full-thickness continuous suturing technique. Another modification was performed by Cordonnier in 1949, who reported a direct anastomosis between the ureter and the bowel mucosa using interrupted sutures by performing approximation of the intestinal serosa to the ureteral adventitia.,

Rectal and recto sigmoid bladder maneuvers were introduced in 1894 and 1895. Giordano and Mauclaire, respectively, introduced the implantation of the ureters into an isolated rectum with construction of abdominal colostomies in both experimental animals and cadavers. In fact, these techniques were applied clinically with minimal success by Remedi in 1905, Kronig in 1907, and Rovsing in 1916. Then in 1952, Boyce and Vest performed a two stage technique for developing a rectal bladder in exstrophy patients. ${ }^{2,5}$ The conduit pathway started by the use of gastro-intestinal segment as a conduit to convey urine outside. In 1956, Edwin Sinaiko was the first to use a gastric segment composed of the antrum of the stomach to create a conduit after performing a significant number of experimental gastric conduits. ${ }^{6}$ In 1935, Seiffert performed the jejunal conduits in two patients and after this the jejunal conduit became a popular technique for urinary diversion in the 1960s passing parallel with the use of radiotherapy for pelvic malignancies. Both jejunal and transverse colon conduits were used because of these segments of bowel were less likely to have been damaged during radiotherapy. ${ }^{7}$ The ileal conduits were performed in 1911 by Zaayer when he published his results of these ileal conduits in two patients and Eugene Bricker was responsible for the introduction of the refinement and repopularization of the ileal conduit technique. In the 1960s and 1970s, Wallace introduced his modification for the ileal conduit by describing a technique of uretero ileal anastomosis using a minimum of sutures resulting in decreased rate of uretero ileal 
anastomosis stricture. ${ }^{1,2,8}$ In 1940, Bricker used the sigmoid colon as a conduit in four patients and placed the urinary stoma near the opened colostomy stoma while Turner-Warwick in 1959 refined Bricker's technique by placing the sigmoid colostomy in the left abdomen and the sigmoid urinary conduit in the right abdomen as a one stage procedure. $^{9}$

In 1977, Rudick and his associates succeeded to develop a continent urinary reservoir using stomach in dogs where they constructed a pouch using a large wedge of gastric fundus and implanted the ureters using a modification of the Paquin technique. ${ }^{10}$ Continent urine reservoirs made from small bowel helped creation of a lowpressure continent bladder. In 1967, Kock and associates developed a low-pressure ileal pouch for patients subjected to proctocolectomy with fecal ileostomies and in 1972 they performed an intussuscepted ileal nipple valve in the fashion initially described by Watsuji in 1899 for gastrostomies. ${ }^{2,11}$ Abol-Enein and Ghoneim developed their novel technique for uretero ileal reimplantation in 1994 to avoid the pitfalls of the intussuscepted ileal vale. Their T-pouch was constructed from $44 \mathrm{~cm}$ of ileum with a second, separate proximal $8-10 \mathrm{~cm}$ segment of ileum for the anti reflux limb. ${ }^{12}$

Regarding orthotopic bladder replacement, the first report of a successful neobladder was published in 1888 by Guido Tizzoni and Alfonso Poggi who performed a two-stage technique using small bowel in a dog. In the first stage they isolated $7 \mathrm{~cm}$ segment of lavaged small bowel which they united in a loop. One month later they anastomosed the ureters to the segment and the segment to the neck of the bladder. In 1912, Georges Lemoine performed total cystectomy with reimplantation of the ureters into the rectum in a man with bladder cancer. However, because of recurrent pyelonephritis, he subsequently displaced the rectum forward and anastomosed it to the urethra, with the sigmoid colon brought down to the anal canal. Although the patient died of postoperative sepsis on the eighteenth day, Lemoine may be credited as the first to perform human orthotopic bladder substitution. ${ }^{2,13}$

In 1951, 40years following Lemoine's rectal neobladder, Couvelaire reported the first use of ileum for complete bladder substitution after cystectomy by performing ileo-prostatic urethral anastomosis at the antimesenteric border and brought the other end of the loop to the skin as a safety valve. ${ }^{2,14}$ Camey of Paris is thought by many to have been responsible for the popularization of total bladder replacement using ileum. He performed the so-called Camey I ileal neobladder in 1959. ${ }^{15}$ In 1987, Hautmann and associates described the creation of the ileal neobladder using a $60-70 \mathrm{~cm}$ segment of distal ileum which was detubularized in a $\mathrm{W}$ or $\mathrm{M}$ shape, anastomosed to the urethra, and the ureters sewn into the reservoir as tunneled implants. ${ }^{2,14,16}$ In 1987, Ghoneim Kock and associates of Mansoura (Egypt) and Goteborg, (Sweden) reported their excellent results in 16 men undergoing cystoprostatectomy, where they performed a modified version of the Kock ileal continent diversion. ${ }^{1}$

In 1965, Hradec and Gil-Vernet individually published their techniques in performing ileocecal neobladders. ${ }^{18,19}$ In 1975, Khafagy and associates of Cairo (Egypt) published their data regarding ileocecal orthotopic bladder replacement following radical cystectomy in 10 patients with carcinoma of the bilharzias bladder. They described the technique of anastomosing the lower part of the cecum to the prostatic urethra or bladder neck, while the ureters were implanted in the ileum (again allowing the native ileocecal valve to reduce reflux). By 1987, they had performed this procedure on 130 patients. ${ }^{20}$

\section{General paradigms in urinary diversion}

\section{Background}

The techniques of urinary diversion after cystectomy have evolved from simple diversion to functional and anatomic restoration aiming at resembling the natural preoperative state. This evolution of urinary diversion has developed along three distinct pathways: conduit, pouch, and neobladder orthotopic reconstruction. The main goal of patient counseling about the type and technique of urinary diversion should be designed to determine the safest method for cancer control, with the least complications over both short and long postoperative follow-up periods, easiest comfortable adjustment for a much better patients' lifestyle, to obtain the best patients' quality of life. ${ }^{21}$ Acceptance of cystectomy and the resulting need for urinary diversion is easier for both patients and clinicians if the burden of an extracorporeal urine sack and lost sexuality can be avoided however this is not assured after orthotopic diversion. ${ }^{22}$ Bladder cancer, neurogenic bladder dysfunction, detrusor muscle over activity and chronic inflammatory diseases of urinary bladder such as interstitial cystitis, tuberculosis and schistosomiasis are main causes for improving or replacing the function of the urinary bladder. ${ }^{23}$

\section{Criteria of patients selection}

The choices of urinary diversion may be limited by either patient or medical criteria or even both. Important patient criteria are patient's preference, age and comorbidity, body mass index (BMI), motivation. Medical criteria which possibly add limiting choices of type of urinary diversion are kidney function, upper urinary tract status, underlying primary disease, indication for cystectomy and limitations concerning the gastrointestinal tract. Other medical criteria are concerning with the status of urethra, sphincter involvement and the patient's ability and motivation to perform intermittent self-catheterization. ${ }^{24}$

Bissada in 1993 highlighted attributes for all continent urinary diversions such as construction of adequate volume, low pressure reservoir with high compliance, reliable continence mechanism, prevention of intestinal ureteric reflux or stenosis, simplicity in construction, avoidance of use of synthetic material, avoidance of the use of excessive lengths of bowel. Also ease of catheterization, avoidance of revision surgery and good cosmetic appearance should be put in mind. ${ }^{25}$ Continent urinary diversions are usually divided into three major categories: procedures including ureterosigmoidostomy and allowing the excretion of urine by evacuation; orthotopic voiding pouches in patients with an intact sphincter mechanism. And the third group includes continent diversions that require catheterization to empty the created reservoir. ${ }^{26}$

\section{Indications}

Specific urinary diversion should be performed on the basis of three important pre operative parameters. The first is mental status of the patient, then his renal function and the third is the overall health. In case of invasive bladder tumor, the choice for construction and replacing the lower urinary tract function usually vary between conduit diversion, bladder replacement or continent diversion..$^{21,24,27}$ In patients suffered from neurogenic bladder dysfunction due to congenital or acquired disorders as in cases of neural tube defect and spinal cord injuries, the main indications for urinary diversion or bladder construction are represented by intractable incontinence, deteriorating renal function and high bladder pressures. The choices 
would include reconstruction of the urinary bladder, replacement or continent diversion. ${ }^{21,24}$ Also, patients suffering from idiopathic interstitial cystitis with failure of all the conservative treatments, the surgical choices range from ileal conduit diversion to orthotopic neobladder reconstruction. ${ }^{27}$

\section{Contraindications}

A. Absolute contraindications: Compromised renal function is an absolute contraindication to continent diversion of any type and this compromised renal function results from long standing obstruction or chronic renal failure with serum creatinine levels 150 to $200 \mu \mathrm{mol} / \mathrm{L}$. Also, severe hepatic dysfunction is also considered as a contraindication to perform continent diversion. In case of inflammatory bowel disease, it may be better to perform an incontinent bowel conduit. Orthotopic bladder reconstruction is considered as absolutely contraindicated in all patients in whom simultaneous excision of urethra is indicated according to their primary tumor. ${ }^{21,22,26}$ The author referred here to two international conferences;

a) The consensus conference convened by the World Health Organization (WHO) and the Society Internationale d'Urologie (SIU) met to critically review reports of urinary diversion and the evidence-based recommendations for urinary diversion that were prepared. ${ }^{21}$

b) The 2nd International Consultation on Bladder Cancer recommendations on the reconstructive options after radical cystectomy, their outcomes, and their complications. ${ }^{28}$

B. Relative contraindications: Mental impairment is considered a relative contraindication when the individual lacks the understanding with regard to new required voiding techniques..$^{27}$ Controversy exists in patients with multifocal, high-grade transitional cell carcinoma and also in patients with multifocal carcinoma in situ. Recurrence in the preserved urethra after cystectomy has been reported to reach up to $12 \%$ with most recurrences noted in patients whose cystectomy specimen showed carcinoma in situ. ${ }^{6}$

\section{Patients satisfaction and quality of life}

Preserving the function of the bladder after radical excision still continues to elaborate great controversy. None of the present surgical options is perfect and each one of these techniques has welldocumented both advantages and disadvantages. ${ }^{29}$ Urinary diversion itself rather than absence of the urinary bladder through cystectomy has the greatest impact on quality of life (QOL) in patients with bladder cancer. The effect of urinary diversion is most profound in terms of sexual function and problems with the diversion technique itself. The basic assumptions gained from previous studies are that patients with continent cutaneous or orthotopic diversions may have an improved QOL compared to those with incontinent ileal conduit urinary diversions. ${ }^{30}$ Still there is lack of a universal agreement upon definition of the term quality of life. WHO has defined QOL as an individual's perception of their position in life in the context of the culture and value system in which they live and in relation to their goals, expectations and standards and concerns? This definition is a broad term and ranging concept affected in a complex way by the physical health of the person, patient's psychological state, and level of independence, social relationships and the relationship to salient features of his environment. ${ }^{31}$
QoL is defined as a module with dynamic multidimensional construct that changes over time and has the ability to be modified and manipulated according to patient's age and experience of both patients and healthcare providers. Health-related quality of life (HRQoL) is defined as a form QoL used for assessment of the impact of illness on subject's life and is considered an important component of survival. The range of parameters assessed by HRQoL measures includes physical function, symptoms, global judgment of health, psychological and social well-being, cognitive functioning, role activities, personal constructs, and satisfaction with care. ${ }^{29,32}$ Acceptance of stoma and rehabilitation in case of ileal conduit diversion varies widely but generally it can be said that urostomy is not an invalidizing operation and psychosocial reintegration is possible and adequate preoperative information particularly on sexual status is very important. Regarding the postoperative status, patients with an ileal conduit usually have the poorest self-images, as defined by a decrease in sexual desire, and all forms of sexual and nonsexual physical contact. ${ }^{32,33}$

The advantages of the various forms of continent urinary diversion are the presumed improvement in QoL compared with conduit diversion. However, continent urinary diversion may have some disadvantages as well. From the technical point of view, continent forms of diversion are more challenging and more time consuming and postoperatively, patients leave the hospital with indwelling catheters. Once the catheters are removed, patients must undergo a period of education in the techniques required to properly care for the reservoir or neobladder. Patients are also at a higher risk for diarrhea and vitamin B12 malabsorption. However, it is generally believed that the QoL advantages of continent urinary diversions outweigh the potential disadvantages. ${ }^{34,35}$

\section{Measurement of QOL}

There are also three symptom scales of fatigue, nausea/vomiting and pain, and six single items that deal with dyspnea, insomnia, appetite loss, constipation, diarrhea, and financial difficulties caused by the disease itself or its treatment. ${ }^{34}$

General: The main parameters for scoring of QoL are the feasibility of bathing, sexual desire, and desire to void like preoperative status and these parameters actually affect the psychological, social and sexual status. ${ }^{33}$ QoL may be measured in clinical practice for several reasons: to assess the impact of the current medical or surgical intervention on QoL, to assess the relationship between QoL and prognosis of these interventions or health outcome, to improve communication for risk identification, to identify preferences, and for use in clinical audit and in clinical governance. ${ }^{36,37}$ QoL tools are important patient-reported outcome measures and useful in achieving or optimizing patientcentered care. ${ }^{28}$ A number of studies have addressed QoL issues following radical cystectomy with various types of urinary diversions and have made comparisons of the impact of different types of urinary diversion on patient QoL. ${ }^{38}$ A major obstacle is lacking of diseasespecific QoL instrument, which universally compares patients after urinary diversion. In addition, the concept of QoL differs significantly between cultures, countries, and races. ${ }^{33}$

One major problem in comparing the results of different studies is lacking of the universal standard questionnaire for the purpose of tracing different domains of QoL in patients subjected to radical cystectomy. The problems include lack of a standard definition of QoL, and cultural differences in discernment and expression of both physical and emotional health. In addition, study sample size as well 
as design and patients characteristics are also confounding factors. The potential effects of sociocultural settings on the concept of QoL have also been raised. The cultural pattern of the studied population can affect the study results. ${ }^{33,39}$

\section{Tools of QoL measurement}

a. Functional score assessment: In general, the functional score assesses the person daily activities. Radical cystectomy is usually considered a debilitating procedure post-operatively; however, some patients may develop their own coping mechanisms and their functional scores return to the baseline pre-operative levels after approximately within one year. Like any major abdominal surgery, muscle and fascial strength would never be compatible to the pre-operative period. Overall, patients learn to protect themselves and are functional post-operatively without any major restriction after a year. ${ }^{40}$ The Functional Assessment of Cancer Therapy-Bladder Cancer (FACT-BL) is a questionnaire specifically designed for patients with bladder cancer. It consists of two parts: a general version (FACT-G), which has been validated and tested for patients with several types of cancer. ${ }^{39}$ Patients well-being in four domains (physical well-being, social/family well being, emotional well-being, and functional well-being) is assessed, with patients responding to statements on a five-level ordinal Likert Scale, ranging from "not at all" to "very much". Higher scores indicate a better outcome. The second part is a bladder cancer-specific module concerning urinary tract symptoms, intestinal symptoms, and sexual symptoms, with a total of 10 statements, plus two more for patients with a stoma, which were omitted in this study. The questions are scored on a scale from 0 to 4 , with 0 being not at all and 4 , very much. ${ }^{39-41}$

b. Symptom score assessment: The most prominent symptoms patients experienced are fatigue and intra-familial difficulties, whereas after the initial year most of the symptoms experienced are about their social life such as voiding and sexual problems. Symptom score likewise usually improves with time and at the 12th-month period return to acceptable values. The major symptoms that are consistent all through the follow-up period affected family and may include sexual problems with the partner and social life due to urinary problems. ${ }^{21,35,40,41}$

c. General health status assessment: General health status scoring actually summarizes the reflection of the previous two scores on the patients' QOL; the functional and the symptoms scores. Likewise, as would be expected, as the functional score of the patients improved and the symptoms decreased, patients' self-reported general health status also improved. ${ }^{41}$

d. Beck's Depression Inventory assessment: There is usually a negative feedback effect between depression and functional score as well as the general health status assessment but a positive feedback between depression and symptom score. Symptom score curve runs parallel to the depression curve. Pre-operatively, the anxiety of losing a functional organ as well as the fear of death due to the cancer causes depressive aspects. Both psychological and health-related QOL measures come to baseline values and stabilize usually after the 12 th-month period, suggesting that the time frame for the adaptation of patients is 12 months after radical cystectomy. Quality of life assessment studies should give an insight to the patients about their long-term outcome; provide the physicians an understanding of their patient follow-up and adaptation. ${ }^{40,41}$ e. Hospital Anxiety and Depression Scale: The Hospital Anxiety and Depression Scale (HADS) comprise 14 items; seven referring to anxiety and seven to depression. It has been validated and tested for reliability. The effectiveness of this scale for detecting mood disorders has been assessed in various cancer populations. The statements are affirmed or rejected on a Likert scale with four options (0 to 3), giving a possible maximal score of 21 for anxiety and depression. Scores greater than 10 indicate a probable anxiety or depressive disorder. ${ }^{39}$

f. QoL \& forms of urinary diversion: The perceived advantage of the various forms of continent urinary diversion is a presumed improvement in quality of life compared with conduit diversion. However, these continent forms of urinary diversion may have some disadvantages as being technically more challenging with more time consuming. ${ }^{36}$ Previous studies demonstrated that patients with continent urinary diversions had better outcomes in terms of different domains of QoL, including sexual functioning, psychological status, and global satisfaction rate. ${ }^{31,33,35}$ Postoperatively, patients are discharged with indwelling catheters. Once the catheters are removed, patients must undergo a period of education in the techniques required to properly care for the reservoir or neobladder. Patients are also at a higher risk for diarrhea and vitamin B12 malabsorption. However, it is generally believed that the quality of life advantages of continent urinary diversions outweigh the potential disadvantages. ${ }^{35}$

Without randomized clinical trials or well-designed prospective longitudinal studies, the optimum form of urinary diversion remains unknown. Both continent and incontinent diversions are available for urinary reconstruction. Orthotopic neobladders optimally preserve the body image, while continent cutaneous diversions represent a reasonable alternative. Ileal conduits represent the fastest, easiest, least complication-prone, and most commonly performed urinary diversion. The isolated segment of ileum used for diversion in ileal conduits is exteriorized through the abdominal wall, where it serves as a passive conduit for urine to drain into an appliance. The presence of a stoma and external appliance can negatively affect a patient's body image. ${ }^{42,43}$ Continent cutaneous reservoirs use a low-pressure pouch constructed of detubularised bowel with a functional mechanism designed to prevent involuntary efflux of urine flow. Reservoirs differ based upon the type of valve mechanism constructed, the type of catheterizable stoma created, and the exact segment of intestine used. The most obvious advantage for this diversion lies in its continent nature, dispensing with the need for an external appliance. However, it requires clean intermittent self-catheterization through the stoma both to empty the reservoir and irrigate retained mucus. The ability of the patient to be technically able to catheterize the stoma over a lifetime cannot be overemphasized. Although these diversions have been largely supplanted by the orthotopic neobladder, they are still used when continence is desired in the setting of non-functional urethra or a positive intra-operatively urethral margin obviating the ability to perform orthotopic diversion. ${ }^{11,33,42,43}$

\section{Complications of urinary diversion}

These complications are divided into two main groups; early and late. Early complications usually occur in approximately $10 \%$ of patients and are wound infection, uretero ileal leakage, intestinal obstruction, intestinal fistula and acute pyelonephritis while late complications occur in $10-20 \%$ of patients and include metabolic 
disorders, stromal stenosis, chronic pyelonephritis, and formation of calculi. ${ }^{27}$

\section{Perioperative and surgery-related complications}

Evaluating the 90-day postoperative period complications instead of the 30-day postoperative period allows the natural evolution of complications over time to be well-recorded and helps identifying modifiable factors that can be targeted for quality improvement. ${ }^{42}$ The complications are usually specific to the type and technique of diversion and are generally categorized into three main categories; those related to the bowel anastomosis, related to the type of reservoir or conduit, and the 3rd are associated with the ureteroenteric anastomosis. Primary complications are those directly attributable to the cystectomy itself, including gastrointestinal complications and secondary complications are those occurring distant from the surgical field, in an organ system other than the urinary tract and postoperative infections. ${ }^{21,42}$ The overall rate of complications associated with the use of conduits is usually similar in case of ileum and colon segments used among properly selected patients. The four most common known complications are pyelonephritis, ureteric obstruction, formation of urinary calculi and stoma-related complications..$^{1,2,43}$ In case of continent urinary diversions, the complications differ between the early postoperative periods compared with the long-term follow up periods and are specific to the type of reservoir. Continent urinary diversions usually show a higher incidence of urinary leaks from the anastomotic lines in the early postoperative period and may be complicated by pouch rupture. This pouch rupture is initially treated with conservative measures, such as catheter drainage or even placement of nephrostomy tubes. If these measures failed, surgical revision of the urinary diversion can then be performed. ${ }^{44}$

\section{Long-term complications}

The important long-term complications are kidney function, stomarelated, problems, bowel-related problems, metabolic disorders, recurrent infections such as pyelonephritis or urinary tract infections and stone formation. ${ }^{42,43}$

\section{Functional complications}

i. Renal function: Absorption of constituents of urine through the bowel mucosa can cause an increase in acid load. The larger the bowel surface used in urinary diversion and the greater the contact time with urine the greater the acid load will be, thus logically continent reconstruction has greater potential of the problem than conduit diversion. Neobladder construction or continent cutaneous reservoir patients with normal renal function are able to compensate for an increased acid load, provided the reservoir is evacuated at regular intervals. In case of renal impairment, the patient's ability to deal with the increased acid load is reduced leading to metabolic acidosis. ${ }^{44,45}$ Normal urothelium has several defense mechanisms against bacteria and the voiding function is a primitive but as effective as a physical action to wash out bacteria. Also, urothelium has its inhibitory action against bacterial adherence while bowel epithelium lacks this inhibitory action; this is one of the reasons why bacteriuria frequently occurs when bowel is used as urinary diversion. Acidic or high osmolar urine itself also has some antibacterial actions. The immune system components whether specific and nonspecific, such as polymorph nuclear leukocytes or secreted immunoglobulins play a role. In patients with urinary diversion, some of these mechanis$\mathrm{ms}$ are impaired and are susceptible to bacterial infection. Several types of urinary diversion may be attained, and the characteristics of urinary tract infection vary according to each type of urinary diversion. ${ }^{21,43}$

ii. Hepatic Function: Infection with Proteus and Klebsiellaspp which are urea-splitting micro-organisms increases the ammonia load in an acute phase. In addition, endotoxins released by Proteus and Klebsiellaspp and others significantly affect hepatic functions regarding transport and metabolism. When intestine is interposed in the urinary tract, absorption of ammonia into the portal circulation are markedly increased owing to the increased load of ammonia from the urine. The normal liver can clear the increased ammonia load, resulting in marked increase in its serum concentrations. It is stated that ammonia is incorporated into the Ornithine cycle for creating urea and it has been shown that the liver rapidly adapts for this incorporation by increasing its capacity for ureagenesis. ${ }^{21,43}$ However, small amounts of endotoxins can significantly affect hepatic metabolism and transport. Hyperammonemic encephalopathy has been reported in patients subjected to ureterosigmoidostomy while patients who have ammoniogenic coma with clinically normal liver function generally have a significant infection with the urease-producing organisms; Proteus and Klebsiella spp. Direct access of bacteria and endotoxins to the liver via the portal circulation results in altered hepatic metabolism without significant alterations in serum levels of hepatic enzyme concentrations. ${ }^{43,46}$ The occurrence of these complications is extremely rare in patients with non-continent diversion such as an ileal conduit. ${ }^{45}$

iii. Drug pharmacokinetics: Many drugs are normally secreted as the changed or unchanged forms in the urine and absorbed by the diverted part of intestinal tract. These absorbed drugs are most likely to cause systemic problems..$^{21}$ The re-absorption of absorbed drugs can result in both diagnostic and therapeutic problems. For example, in patients with diabetes, urine glucose testing is inaccurate because of glucose re-absorption from the intestinal mucosa. To reduce the chance of reabsorption in patients who are planned to receive chemotherapy, they should have a catheter placed during treatment to allow frequent evacuation of urine. Other drugs including several antibiotics, Methotrexate, Phenytoin, Theophylline and Lithium, are known to be absorbed by the diverted intestinal segments. $^{44,45}$

iv. Bone: As a result of long-term effect of urinary intestinal diversion, bone demineralization usually occurs and is clearly demonstrated in children with rickets and adults with osteomalacia especially after ureterosigmoidostomy. In these conditions bone mineral loss is replaced by osteoid tissues with decreased bone strength. ${ }^{42-45}$ The resultant chronic acidosis may affect the skeleton in three ways. The first is shown when bone minerals, including calcium, carbonate and sodium, act as buffers in exchange for hydrogen ions, thus decreasing the skeletal calcium content. Secondly in the kidney, acidosis impairs hydroxylation of 25-hydroxycholecalciferol and activated vitamin D deficiency results in bone mineralization defects. The third way is clearly obvious when acidosis activates osteoclasts cells with the result of bone resorption. In addition following ileal resection, poor intestinal absorption of calcium and vitamin D may occur. ${ }^{47}$ Patients with preexisting renal disease are prone to acidosis and then impaired activated vitamin $\mathrm{D}$ production secondary to renal tubular cell damage. Clinically, these patients may be symptom-free, complain of pain due to involvement of the weight bearing joints or present with pathological fractures. Labo- 
ratory tests may be within normal values despite symptoms, although in general there is reduced serum calcium and phosphate with elevated alkaline phosphatase..$^{21,42,43,47}$ Imaging studies are usually normal and bone densitometry is useful but may need repeated testing. Bone biopsy may be the only way to confirm diagnosis. ${ }^{47-49}$

i. Bowel function: A major concern regarding urinary diversion when intestinal segments are used during reconstruction of any form of urinary diversion is the subsequent effect on bowel function. Patients undergoing a urinary diversion often complain of diarrhea which may be intractable where the resection of the terminal ileum carries the risk of reduction in both bile salt and fat absorption. ${ }^{45}$ Following the use of a relatively long segment of intestine to create a diversion and accordingly the loss of intestinal absorptive surfaces, a number of nutritional complications can also occur. The creation of ileocecal bladder with removal of the terminal ileum or the ileocecal junction can interfere with the absorption of bile salts and may lead to the colonization of bacteria with increasing the bacterial load within the terminal ileum leading to chronic diarrhea. According to this fact, the terminal ileum is not recommended to be used during urinary diversion to avoid the occurrence of this side-effect as well as Vitamin B12 deficiency. ${ }^{21}$ It is well-known that bile salts are reabsorbed and recycled at the terminal ileum and are essential for both fat digestion and the uptake of fat-soluble vitamins $\mathrm{A}$ and $\mathrm{D}$. In case of construction of urinary diversion using the ileocecal junction, larger quantities of bile salts and un-metabolized lipids enter the colon directly due to removal of the terminal ileum causing colonic mucosal irritation and steatorrhea. ${ }^{42,43,47}$ The use of colonic segment for constructing urinary diversion and colonic resections may lead to diarrhea, dehydration and acidosis when the shortened colonic segment does not coupe with the absorption of the alkaline ileal contents during urinary diversion. ${ }^{48}$

ii. Metabolic consequences: Long-term follow-up of urinary diversion usually focuses on two important aspects; metabolic aspects and specific complications of different types of urinary diversions. ${ }^{48}$ Metabolic complications can be minimized by careful patient selection, choosing of the appropriate bowel segment, and meticulous and strict follow-up. There are important measures that should be taken to prevent metabolic dysfunction. These measures are keeping good renal function, regular voiding to completion, sterile urine and normal blood gas analysis. ${ }^{44,45}$

iii.Hyperchloremic acidosis: Hyperchloremic acidosis is prevalent well-known clinical finding following ureterosigmoidostomy where urine comes in contact with the whole colonic mucosa. However, chloride absorption and bicarbonate excretion are more pronounced in the colon, and there is evidence suggesting that inherent chloride absorption is maintained when in long contact with urine. To reduce the risk of hyperchloremic acidosis particularly in patient with renal impairment, ileum should be used rather than colon for bladder reconstruction. ${ }^{21,42-45}$ However, in patients having undergone construction of ileal conduit diversion, mild to moderate acidosis could be happened up to $15 \%$. In patients with a continent urinary diversion, logically the time of contact of urine is markedly longer and the exposed surface area of bowel mucosa to urine is much larger and consequently this longer contact can lead to a higher incidence of electrolyte disturbances with metabolic acidosis and may reach up to $50 \%$ of patients. ${ }^{48}$ In these patients, the elevated concentration of serum chloride is associated with a decrease in base excess. Therefore, treatment of hyperchloremic acidosis consists of administration of alkalizing agents. Prophylactic alkali substitution should be commenced at a base excess below- $2.5 \mathrm{mmol} / \mathrm{L}$, aiming at avoiding the long-term complications of clinically evident acidosis. ${ }^{21}$

iv. Electrolyte abnormalities: Hypokalemia, hypocalcaemia, and hypomagnesaemia are the electrolyte abnormalities that may be associated with urinary diversion. Hypokalemia and total body depletion of potassium may be seen with ileal and but more frequently with colonic urinary intestinal diversion as ileal segments can absorb more potassium. Previous reports have shown that ileal segments when exposed to high concentrations of potassium in the urine reabsorb some of the potassium contents, whereas colon is less likely to do so. ${ }^{21}$ Hypokalemia, as an important electrolyte imbalance is caused by both intestinal loss as in chronic diarrhoea and by renal wasting. However, hypocalcaemia in patients with urinary diversion is caused by renal wasting and by depleted calcium stores when the chronic metabolic acidosis is tried to be buffered by bone carbonate. Therefore, mobilization of carbonate from bone results in calcium release from the bone and the presence of acidosis further inhibits calcium reabsorption and this excess calcium is cleared by the kidneys. ${ }^{49,50}$ Hypocalcemia and/or hypomagnesemia are infrequent complications of urinary diversion. As previously mentioned, hypocalcemia is a consequence of depleted body calcium stores and excessive renal wasting and chronic acidosis is buffered by carbonate in the bone, with subsequent release of calcium into the circulation. The kidneys clear the released calcium, resulting in a gradual decrease in body calcium stores. Magnesium deficiency is usually due to nutritional depletion, but it may result from renal wasting. Altered calcium metabolism, acidosis, and sulfate metabolism all interfere with renal tubular magnesium reabsorption. . $^{21,48-50}$

\section{Obstructive complications}

I. Ureteroenteric stenosis: The incidence of secondary ureteroenteric anastomosis stricture after urinary diversion ranges that may reach up to $14 \%$ and ureterosigmoidostomy carries the highest rate of stenosis reported reaching up to $22 \%$. These strictures at the ureteroenteric anastomoses are candidate for numerous complications, including recurrent urinary tract infection, sepsis, stone formation, and renal failure that is often clinically silent. ${ }^{51}$ The management of this ureteroenteric stenosis presents a particular challenge for the urologists. The standard and most effective treatment protocol of these strictures is usually open surgical repair for ureteral reimplantation with overall success rate up to $80 \%{ }^{42,43}$ However, this open surgical repair is an invasive procedure and with difficult techniques that carry considerable morbidity and prolonged hospitalization however the endoscopic interference has been established as alternative treatment with less invasive approach. ${ }^{51}$ The ureteroenteric anastomosis strictures and stenosis are the most frequent causes of gradual deterioration of renal function after urinary diversion due to complete luminal obstruction with consecutive progressive hydronephrosis. ${ }^{52}$ There are two main causes of occurrence of ureteroenteric anastomosis strictures, contact of the intestinal and ureteral mucosa and technical defects causing ureteral ischemia. Moreover other causes of occurrence of ureteroenteric anastomosis strictures have been identified as predisposing factors. The tissue incompatibility causes transitory epithelial metaplasia leading to ureteral cicatrization and stenosis, extravasation of urine and infections. ${ }^{53}$ There are important technical factors should be established for avoiding ureteroenteric stenosis. These technical factors include meticulous surgical techni- 
que involving mobilization of the sigmoid mesentery cephalad to the origin of the inferior mesenteric artery to avoid obstruction of the left ureter, and preservation of the adventitia of ureters to optimize blood supply and the use of soft stents postoperatively. ${ }^{51}$

II. Urinary retention: Voiding dysfunction after orthotopic neobladder construction can be divided into two main groups of dysfunction; failure of the bladder emptying whether complete or incomplete and failure to store urine. Patient with failure to store urine usually reports this complaint during the daytime, night-time or both. Regarding improvement of quality of life in patients experiencing voiding dysfunction, the orthotopic neobladder has lower score compared to the ileal conduit. Comparing of voiding dysfunction as regard the severity and prevalence of in many surgical series is difficult according many factors of assessment such as variability in end points, definitions, length of follow-up, patient age, patient sex, and surgical technique. ${ }^{42,43,54}$

Retention of urine is much more common in women than men after orthotopic reconstruction and this retention might occur early but often presents after years of good function and emptying of the neobladder. The etiology of urinary retention in women showed much debate and most authors believe that it is due to a mechanical kink in the anastomoses between urethra and reservoir. Lateral straining cystogram reveals the full pouch falls posteriorly during the Valsalva maneuver in some patients. ${ }^{42,43,55-57}$

Other suggested points for explaining the etiology of urinary retention include autonomic denervation of the urethral remnant during surgical dissection and disordered re-innervations resulting in the inability to relax the urethral sphincter during the act of voiding. ${ }^{42,55-58}$ Urinary retention is treated with intermittent and regular catheterization; while alpha-blockers seem ineffective so every women undergoing neobladder reconstruction should be advised that intermittent catheterization might be required for adequate emptying of their neobladders. Transurethral resection of a urethral fold and open reduction of the pouch size with anterior fixation to the abdominal wall have also been described. ${ }^{55}$

Oncologic complications: The risk of disease relapse following radical cystectomy is reported by many investigators to reach as high as $70 \%$, with the majority occurring within two years of surgery. ${ }^{59}$ Following cystectomy and urinary diversion, the disease recurrence is in the form of local, distant, and with both local and distant recurrences. ${ }^{60}$ Patients with $\mathrm{T} 2$ disease usually have a significantly longer time to recurrence compared with patients with $\mathrm{T} 3$ or $\mathrm{T} 4$ disease. The most common pattern of recurrence is usually pelvic lymphadenopathy. Local pelvic recurrence may appear as a soft tissue mass, which involved muscle, urethra or vagina. Sites of distant metastasis usually include the liver, bone and lungs. Liver metastasis may be associated with nodal disease in the majority of disease recurrence. Imaging studies are of important role in detecting and defining the sites of recurrent disease. However, familiarity with any changes of post-surgical status and the normal appearances of the urinary diversion procedures is crucial to the interpretation of imaging studies. ${ }^{59}$

\section{Conclusion}

Many decades ago, the techniques of urinary diversion have evolved from simple diverting techniques through a conduit to the orthotopic neobladder reconstruction with efforts to provide an improved QoL. Since the 1950s, the further evolution of lower urinary tract reconstruction has developed along many distinct paths until reaching the most recent form of diversion to the native, intact urethra; neobladder. Patient counseling for better satisfaction about urinary diversion should be tailored to determine the safest method for cancer control, with the fewest complications over both the short and the long term, and the easiest adjustment for patients' lifestyle, thereby supporting the best patients' quality of life. The choices of diversion may be limited by either patient or medical criteria or even both. HRQoL measures should include physical function, symptoms, global judgment of health, psychological and social wellbeing, cognitive functioning, role activities, personal constructs, and satisfaction with care.

\section{Acknowledgements}

None.

\section{Conflict of interest}

The author declares no conflict of interest.

\section{References}

1. Nagele U, Sievert K, Merseburger AS, et al. Urinary Diversion Following Cystectomy. EAU Update Series. 2005;(3):129-137.

2. Webster GD, Andrew C, Peterson AC. History of urinary diversion techniques. In: Kreder KJ, Stone AR, editors. Urinary Diversion. 2nd ed. United Kingdom: Abingdon, Oxford shire, Taylor \& Francis, CRC Press; 2005. p. 1-44.

3. Nesbit RM. Ureterosigmoid anastomosis by direct elliptical connection; a preliminary report. J Urol. 1949;61(4):728-734.

4. Cordonnier JJ. Ureterosigmoid anastomosis. J Urol. 1950;63:276-285.

5. Boyce WH, Vest SA. A new concept concerning treatment of exstrophy of the bladder. J Urol. 1952;67(4):503-517.

6. Sinaiko ES. Artificial bladder from a gastric pouch. Surg Gynecol Obstet. 1960;111:155-162.

7. Golimbu M, Morales P. Jejunal conduits: technique and complications. J Urol. 1975;113(6):787-795.

8. Wallace DM. Ureteric diversion using a conduit: a simplified technique. Br J Urol. 1966;38(5):522-527.

9. Turner-Warwick RT. Technique for the separate diversion of urine and faeces. Lancet. 1959;1(7081):1021-1022.

10. Rudick J, Schonholz S, Weber HN. The gastric bladder: a continent reservoir for urinary diversion. Surgery. 1977;82(1):1-8.

11. Kock NG. The development of the continent ileal reservoir (Kock pouch) and its application in patients requiring urinary diversion or bladder replacement. In: King LR, et al. editors. Bladder Reconstruction and Continent Urinary Diversion. USA: Year Book Medical Publishers; 1991. p. 247-271.

12. Abol-Enein H, Ghoneim MA. A novel uretero-ileal reimplantation technique: A serosal lined tunnel. A preliminary report. J Urol. 1994;151(5):1193-1197.

13. Basic DT, Hadzi-Djokic J, Ignjatovic I. The history of urinary diversion. Acta Chir Iugosl. 2007;54(4):9-17.

14. Hautmann RE. Ileal bladder substitute. Urologe A. 2008;47(1):33-34, 36- 40 .

15. Camey M. Bladder replacement by ileocystoplasty following radical cystectomy. World J Urol. 1985;3(3):161-166.

16. Hautmann RE, Egghart G, Frohneberg D, et al. The ileal neobladder. $J$ Urol. 1988;139(1):39-42.

17. Ghoneim MA, Kock NG, Lycke G, et al. An appliance-free sphinctercontrolled bladder substitute: the urethral Kock pouch. J Urol. 1987;138(5):1150-1154. 
18. Hradec EA. Bladder substitution: indications and results in 114 operations. J Urol. 1965;94(4):406-417.

19. Gil-Vernet JM. The ileocolic segment in urologic surgery. $J$ Urol. 1965;94(4):418-426.

20. Khafagy MM, el-Kalawy M, Ibrahim A, et al. Radical cystectomy and ileocecal bladder reconstruction for carcinoma of the urinary bladder. $\mathrm{Br}$ J Urol. 1987;60(1):60-63.

21. Hautmann RE, Abol-Enein H, Hafez K, et al. Urinary diversion. Urology. 2007;69(1 Suppl):17-49.

22. Burkhard FC, Kessler TM, Mills R, et al. Continent urinary diversion. Crit Rev Oncol Hematol. 2006;57(3):255-264.

23. Cody JD, Nabi G, Dublin N, et al. Urinary diversion and bladder reconstruction/replacement using intestinal segments for intractable incontinence or following cystectomy. Cochrane Database Syst Rev. 2012;2:CD003306.

24. Thuroff JW, Hampel C, Leicht W, et al. Indications for different types of urinary diversion. Urologe A. 2012;51(4):473-476.

25. Bissada NK. Characteristics and use of the in situ appendix as a continent catheterization stoma for continent urinary diversion in adults. J Urol. 1993;150(1):151-152.

26. Moon A, Vasdev N, Thorpe AC. Continent Urinary Diversion. Indian J Urol. 2013;29(4):303-309.

27. Siracusano S, Ciciliato S, Visalli F, et al. Current Trends in Urinary Diversion in Men. In: Bladder Cancer-From Basic Science to Robotic Surgery. In: Abdullah Erdem, editor. Canada: InTech Corporation; 2012. p. $354-360$

28. Hautmann RE, Abol-Enein H, Davidsson T, et al. ICUD-EAU International Consultation on Bladder Cancer 2012: Urinary diversion. Eur Urol. 2013;63(1):67-80.

29. Somani BK, MacLennan SJ, N'Dow J. Quality of Life with Urinary Diversion. Eur Urol Supp. 2010;9(10):763-771.

30. Parkinson JP, Konety BR. Health related quality of life assessments for patients with bladder cancer. J Urol. 2004;172(6 Pt 1):2130-2136.

31. Gerharz EW, Mansson A, Hunt S, et al. Quality of life after cystectomy and urinary diversion: an evidence based analysis. $J$ Urol. 2005;174(5):1729-1736.

32. Varricchio CG, Ferrans CE. Quality of life assessments in clinical practice. Semin Oncol Nurs. 2010;26(1):12-17.

33. Asgari MA, Safarinejad MR, Shakhssalim N, et al. Quality of life after radical cystectomy for bladder cancer in men with an ileal conduit or continent urinary diversion: A comparative study. Urol Ann. 2013;5(3):190-196

34. Saika T, Arata R, Tsushima T, et al. Health-related quality of life after radical cystectomy for bladder cancer in elderly patients with an ileal conduit, uretero cutaneostomy, or orthotopic urinary reservoir: a comparative questionnaire survey. Acta Med Okayama. 2007;61(4):199 203.

35. Porter MP, Penson DF. Health Related Quality Of Life after Radical Cystectomy and Urinary Diversion for Bladder Cancer: A Systematic Review and Critical Analysis of the Literature. J Urol. 2005;173(4):1318-1322.

36. Higginson IJ, Carr AJ. Measuring quality of life: using quality of life measures in the clinical setting. BMJ. 2001;322(7297):1297-1300.

37. Varricchio CG, Ferrans CE. Quality of life assessments in clinical practice. Semin Oncol Nurs. 2010;26(1):12-17.

38. Park J, Ahn H. Radical cystectomy and orthotopic bladder substitution using ileum. Korean J Urol. 2011;52(4):233-240.
39. Mansson A, Al Amin M, Malmstrom PU, et al Patient-assessed outcomes in Swedish and Egyptian men undergoing radical cystectomy and orthotopic bladder substitution: A prospective comparative study. Urology. 2007;70(6):1086-1090.

40. Kulaksizoglu H, Toktas G, Kulaksizoglu IB, et al. When Should Quality of Life be Measured after Radical Cystectomy? Eur Urol. 2002;42(4):350-355.

41. Modh RA, Mulhall JP, Gilbert SM. Sexual dysfunction after cystectomy and urinary diversion. Nat Rev Urol. 2014;11(8):445-453.

42. Hautmann RE, Hautmann SH, Hautmann O. Complications associated with urinary diversion. Nat Rev Urol. 2011;8(12):667-677.

43. Lee RK, Abol-Enein H, Artibani W, et al. Urinary diversion after radical cystectomy for bladder cancer: options, patient selection, and outcomes. BJU Int. 2014;113(1):11-23.

44. Moon A, Vasdev N, Thorpe AC. Continent urinary diversion. Indian J Urol. 2013;29(4):303-309.

45. Vasdev N, Moon A, Thorpe AC. Metabolic complications of urinary intestinal diversion. Indian J Urol. 2013;29(4):310-315.

46. Degener S, Roth S, Mathers MJ, et al. Follow-up care - consequences of urinary diversion after bladder cancer. Urologe A. 2014;53(2):253-264.

47. Mills RD, Studer UE. Metabolic consequences of continent urinary diversion. J Urol. 1999;161(4):1057-1066.

48. Stein R, Rubenwolf P. Metabolic Consequences after Urinary Diversion. Front Pediatr. 2014;2:15.

49. Albersen M, Joniau S, Van Poppel H, et al. Urea-splitting urinary tract infection contributing to hyperammonemic encephalopathy. Nat Clin Pract Urol. 2007;4(8):455-458.

50. Van der Aa F, Joniau S, Van Den Branden M, et al. Metabolic changes after urinary diversion. Adv Urol. 2011:764325.

51. Sataa S, Zrayer O, Mizouni H, et al. Percutaneous Dilatation of Nonmalignant Ureteroenteric Anastomotic Strictures in Patients with Urinary Diversion After Cystectomy for Bladder Cancer:7 patients. Uro Today Int J. 2012;5(6):59.

52. Maksimovic HM, Markovic BB, Pejcic T, et al. Interventional radiology methods of treating ureteral strictures developed on anastomoses after the radical cystectomy in Mainz pouch II and ileal conduit derivation. Acta Chir Iugosl. 2007;54(4):83-87.

53. Touiti D, Gelet A, Deligne E, et al. Treatment of uretero-intestinal and uretero vesical strictures by Acucise balloon catheter. Eur Urol. 2002;42(1):49-54.

54. Steers WD. Voiding dysfunction in the orthotopic neobladder. World $J$ Urol. 2000;18(5):330-337.

55. Ali El Dein B, Gomha M, Ghoneim MA. Critical evaluation of the problem of chronic urinary retention after orthotopic bladder substitution in women. $J$ Urol. 2002;168(2):587-592.

56. Hautmann RE. Urinary diversion: ileal conduit to neobladder. $J$ Urol. 2003;169(3):834-842.

57. Stein JP, Penson DF, Wu SD, et al. Pathological guidelines for orthotopic urinary diversion in women with bladder cancer: a review of the literature. J Urol. 2007;178(3):756-760.

58. Hautmann RE, Volkmer BG, Schumacher MC, et al. Long-term results of standard procedures in urology: the ileal neobladder. World $J$ Urol. 2006;24(3):305-314.

59. Koh DM, Husband JE. Patterns of recurrence of bladder carcinoma following radical cystectomy. Cancer Imaging. 2003;3:96-100.

60. Yang G, Whitson JM, Breyer BN, et al. Oncological and Functional Outcomes of Radical Cystectomy and Orthotopic Bladder Replacement in Women. Urology. 2011;77(4):878-883. 